\title{
Study on the dispersion degree of chopped carbon fibers dispersed in fluid medium by microscopic analysis
}

\author{
Xuanyu Wang ${ }^{\text {a* }}$, Zhilong Liu ${ }^{\mathrm{b}}$ \\ Laboratory of Pyrotechnical Technology, Institute of NBC Defense, Beijing 102205 , P.R.China \\ awxycrs@aliyun.com, bzlliu@aliyun.com
}

Keywords: Carbon fiber; fluid; dispersion; explosion; ultrasonic waves; microscopic analysis.

Abstract. In order to study the dispersion effect of chopped carbon fibers in fluid medium, an experiment was designed to disperse carbon fibers by explosion in the air while another was by ultrasonic waves in mixed solvent of ethanol and acetone. A characterizing method has been set up based on the number of single chopped carbon fibers. For an example, the dimension, shape and distributing law were measured based on the experimental data of explosive dispersion in the air. By the results, the chopped carbon fibers can be effectively dispersed in fluid medium by some outside force. The main procedure of ultrasonic dispersion for chopped carbon fibers in the mixed solvent of ethanol and acetone is single carbon fiber silks separated from each other along radial direction. The main procedure of explosive dispersion in the air is not only single carbon fiber silks separated from each other along radial direction but a little of single carbon fibers are broken into small pieces along axial direction. The least discrete unit is a single carbon fiber silk while the bigger one is an aggregate which is composed of several single carbon fiber silks.

\section{Introduction}

Carbon Fiber (CF) has a lot of good performances, such as high temperature resistance, corrosion resistance, high intensity, big conductance and diathermancy, so it is widely used in many fields [1]. CF may be classed into three kinds according to the type of their original organic silk, which includes polyacrylonitrile-based carbon fiber, pitch-based carbon fiber and rayon-based carbon fiber. Since polyacrylonitrile-based carbon fiber is used as strong reflector of radar waves and the absorber of electromagnetic wave [2], it is widely applied in many high-tech fields, such as aviation, spaceflight, national defence and other fields.

Chopped CF is processed from long CF by cutting machine and is widely applied in various multiple materials when it is symmetrically dispersed into some fluid. According to the patent [3], CF may be dispersed into clouds by explosion to interfere with millimeter wave detector. Jueshi Qian and Congbo Xie have studied the effect of polycarboxylate superplasticizer on dispersion of CF in cement-based material [4]. Ying Cui, Yu Sun and Bo Wu have studied the influence of dispersant coating treatment on the properties of CF dispersion in oily substrate [5]. There is no report about quantitative study except the above qualitative analysis about the dispersion effect for CF in fluid. Therefore, a new method has been set up by microscopic analysis based on the number of single chopped CF to characterize the dispersion effect of chopped $\mathrm{CF}$ in fluid medium.

\section{Experiment}

\section{Reagent and Instrument}

Chopped polyacrylonitrile-based CF: the average length of the chopped CF is $4 \mathrm{~mm}$. The diameter of a single CF silk is $7 \mu \mathrm{m}$. The tensile strength is $3800 \mathrm{MPa}$, the tensile modulus is $228 \mathrm{GPa}$. Fig. 1 is the experimental sample. Fig. 2 is its micro morphology analyzed by HITACHI S-4800 SEM.

AR alcohol 500ml. AR acetone 500ml. 10g TNT. An electric detonator. A simulative bomb with a height $10 \mathrm{~cm}$ and a diameter $5 \mathrm{~cm}$. KQ-250DB ultrasonic producer with an electric power $250 \mathrm{~W}$. BT9300-PLUS microscopic image analyzer with a precision $0.1 \mu \mathrm{m}$. 


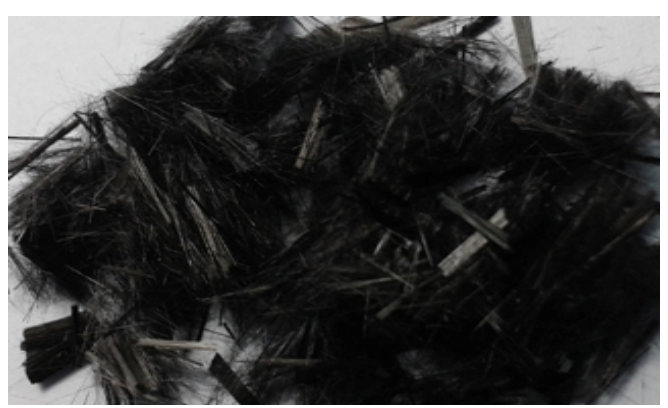

Fig.1. The Chopped CF Sample

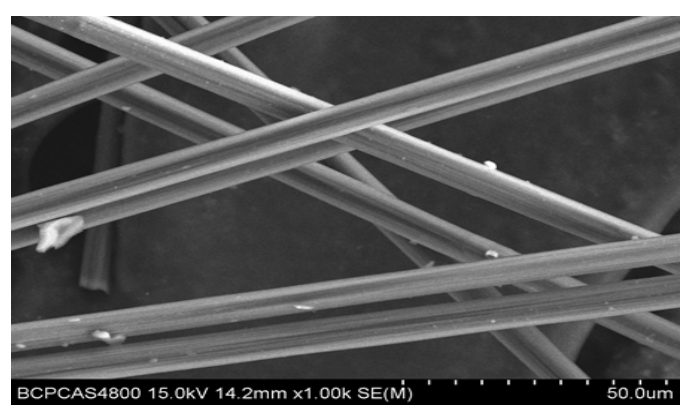

Fig.2. Micro morphology of the chopped CF

\section{Experimental Procedure}

Dispersion in the Air. The simulative bomb was filled by $4 \mathrm{~mm}$ chopped CF, the ratio of TNT to CF was 0.28 , the filling density of the CF was $0.2 \mathrm{~g} / \mathrm{ml}$. The bomb was detonated at a height of $2 \mathrm{~m}$ under small wind speed. Fig. 3 is the sample observed by the microscope.

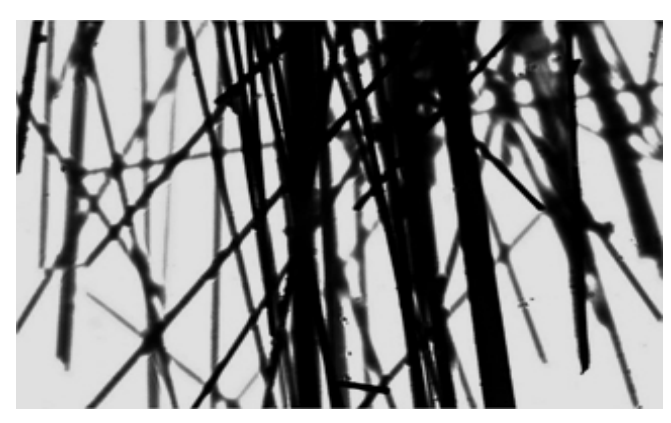

Fig.3. CF dispersed in the air

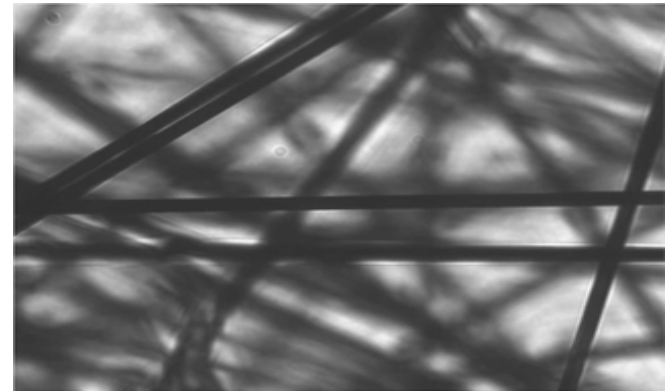

Fig.4. CF dispersed in the solvent

Dispersion in the Mixed Solvent of Ethanol and Acetone. 0.1g Chopped CF was put into 100ml the mixed solvent and acetone at $60^{\circ} \mathrm{C}$. Then it was dispersed for $10 \mathrm{mins}$ by ultrasonic producer after marinated 2h. Fig. 4 is the sample of the chopped CF dispersed in the mixed solvent by ultrasonic waves and observed by the microscope.

\section{Results}

\section{Characterizing Method for Dispersion Degree}

Since the ratio of axial to radial size of a single chopped CF silk is very big, which is $4000 / 7$, the characterizing method for dispersion degree of the CF is very different from ordinary global particles.

The chopped $\mathrm{CF}$ is ordinarily fascicular unit formed by a number of single $\mathrm{CF}$ silks before being dispersed by outside force. The number perhaps is very large, even thousands upon thousands. The function of dispersion is changing the fascicular unit into many single $\mathrm{CF}$ silks or smaller unit composed of several single CF silks.

Since a single carbon fiber silk has a good mechanical performance in radial direction, it is supposed that any radial second-break will not be occurred against a single CF silk.

$\mathrm{CF}$ is brittle and polycrystal and there are a lot of micro-holes and other disfigurements within the original silks [6]. Moreover, the axial size is far larger than radial. As a result, the single CF silk will be broken in axial direction when the shear stress is large enough.

Characterizing for Dispersion Effect in Radial Direction Based on the Number of CF. Fig. 5 is the chopped CF dispersed in the air. According to Fig.5, the smaller discrete units are planar surfaces including 1, 2, 3 or several pieces of single carbon fiber silks.

The radial projective size of a single CF silk is apparently equal to the diameter of a single CF silk while the smaller discrete unit size is approximately equal to the product of the diameter and the number of the CF silks included in it. On the contrary, the number of the single CF silks involved within the discrete unit may be determined by the radial projective size. The smaller the number is, the better the dispersion effect is. Moreover, the least discrete unit is a single carbon fiber silk. 


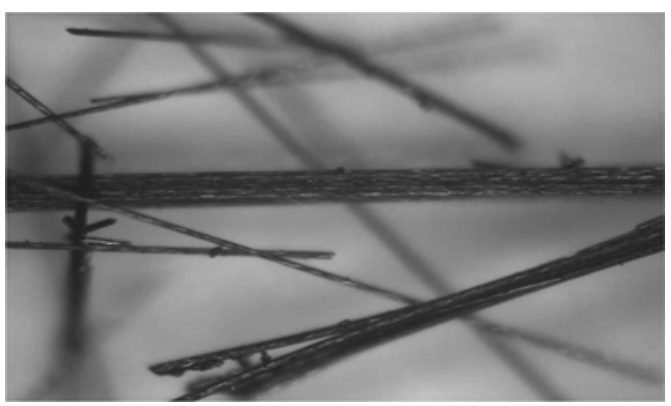

Fig.5. Discrete CF units

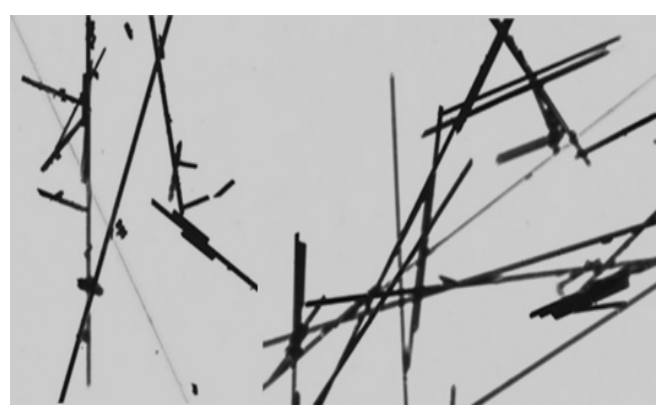

Fig.6. Fractured CF dispersed by explosion

Equivalent Treatment against the Breaking of Single Carbon Fibers in Axial Direction. For an explosive dispersion, the breaking of single carbon fibers is apparent in axial direction and some results are shown in Fig.6. Several broken pieces of CF are equivalently treated as a whole single CF silk. Some ranges in length are determined, such as $(0-0.25],(0.26-0.75]$ and $(0.75-1.0)$, which is to describe and count up the number of the broken pieces of CF with a length of 1/4, 2/4(1/2) and 3/4 of a single CF silk. The equivalent number of the broken pieces correspondingly is $0.25,0.5$ and 0.75 .

\section{Collecting the Statistics of the Experiment}

The dispersion of chopped CF dispersed by explosion in the air has been studied as an example. The radial projective size of a discrete unit is supposed as $W_{i}$, the diameter of a single CF silk is $d_{0}=7 \mu \mathrm{m}$, so the equivalent number of the $\mathrm{CF}$ in the discrete unit may be determined as Eq.1.

$$
n_{e i}=W_{i} / d_{0} \text {. }
$$

The number of the discrete units with a same radial projective size is supposed as $n_{s i}$, and then the total equivalent number $N_{i}$ of the CF silks may be calculated as Eq.2.

$N_{i}=n_{e i} \times n_{s i}$.

10 fields of vision are stochastically selected and analyzed. The results are showed in Table 1.

Table 1 The tested results of the chopped CF sample dispersed by explosion

\begin{tabular}{cccc||cccc}
\hline$n_{e i}$ & $W_{i} / \mu \mathrm{m}$ & $n_{s i}$ & $N_{i}$ & $n_{e i}$ & $W_{i} / \mu \mathrm{m}$ & $n_{s i}$ & $N_{i}$ \\
\hline 0.25 & 7 & 41 & 10.25 & 6 & 42 & 9 & 54 \\
0.5 & 7 & 34 & 17 & 7 & 49 & 6 & 42 \\
0.75 & 7 & 28 & 21 & 8 & 56 & 6 & 48 \\
1 & 7 & 134 & 134 & 9 & 63 & 5 & 45 \\
2 & 14 & 51 & 102 & 10 & 70 & 3 & 30 \\
3 & 21 & 24 & 72 & 11 & 77 & 2 & 22 \\
4 & 28 & 17 & 68 & 12 & 84 & 1 & 12 \\
5 & 35 & 12 & 60 & 13 & 91 & 0 & 0 \\
\hline
\end{tabular}

According to Table 1, the total equivalent number $N$ of the single CF silks in the whole vision may be determined as Eq.3.

$N=\sum N_{i}=737.25$.

\section{Analyzing the Experimental Result}

The distributing frequency of any discrete unit in the dispersion system is determined as Eq.4.

$f_{i}=N_{i} / N$.

The accumulation of distributing frequency of various discrete units is determined as Eq.5. 
$F_{i}=\sum f_{i}$.

The analyzed results are showed in Fig.7 and Fig.8.

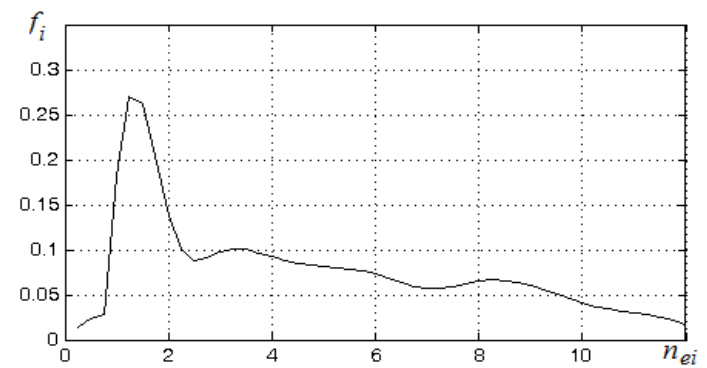

Fig.7. The frequency distributing curve

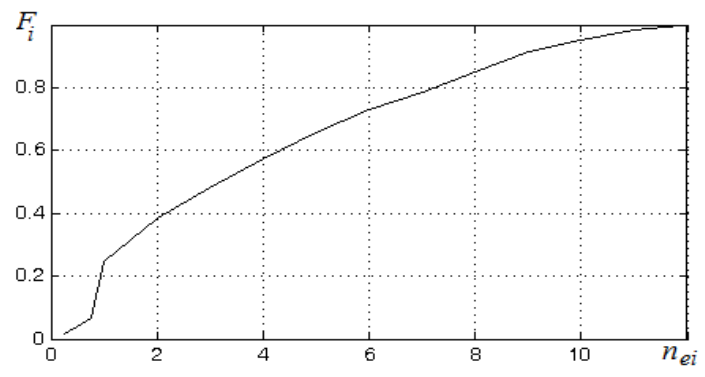

Fig.8. The integral distributing curve

According to Fig.7, the typical equivalent number of the $\mathrm{CF}$ in all discrete units is 1.25 . According to Fig. 8, 50\% of the sample CF are dispersed into small discrete unit composed of 1, 2 or 3 single CF silks, which contains a lot of small pieces produced by breaking of single CF in axial direction.

\section{Conclusions}

The chopped CF can be effectively dispersed in fluid medium by explosion, ultrasonic waves and other outside forces. By the analysis, the main procedure of ultrasonic dispersion for $\mathrm{CF}$ in the mixed solvent of ethanol and acetone is single CF silks separated from each other along radial direction. The main procedure of explosive dispersion for CF in the air not only is single CF silks separated from each other along radial direction but a little of single $\mathrm{CF}$ are broken along axis.

The dispersion effect of chopped CF in fluid medium may be characterized according to the number of single chopped CF silks included in the discrete unit. The least discrete unit is a single CF silk while the bigger one is an aggregate which is composed of several single CF silks. The bigger the discrete unit is, the bigger the number is and the worse the dispersion effect is. Furthermore, the dispersion effect is enhanced by the radial separation while the effective length is decreased by the axial breaking in the dispersing process.

The characterizing method may be applied to describe the dispersion effect of chopped $\mathrm{CF}$ and similar objects in fluid medium, which must have a large ratio of axial to radial size. The characteristic parameters may be determined according to the frequency and integral distributing curve of the sample. Furthermore, the kinetic law and other performances of the dispersion system may be quantitatively studied or described according to the characterizing results.

\section{References}

[1] Lingmei Kong, Wei Zheng, Yanyan Qi, et al. Development of three kinds of high performance fiber [J]. Synthetic Fiber in China, 2013, 42(5): 27-31 (in Chinese).

[2] Hua Qiu, Jihai Tang, Qi Yang, et al. Research development of fiber absorbent [J]. Surface Technology, 2010, 9(6): 66-70 (in Chinese).

[3] Rouse, William G, Connie S, et al. Millimeter wave screening cloud and method [P]. U.S. Patent 5148173, 1992.

[4] Jueshi Qian, Congbo Xie, Haijuan Xing, et al. Effect of polycarboxylate superplasticizer on dispersion of carbon fiber in cement-based material [J]. Journal of Function Materials, 2013,44(16): 2389-2393 (in Chinese).

[5] Ying Cui, Yu Sun, Bo $\mathrm{Wu}$, et al. Influence of dispersant coating treatment on the properties of carbon fiber dispersion in oily substrate [J]. Surface Technology, 2015, 44(1):112-117 (in Chinese).

[6] Yurong Yang, Guomin Yan, Li Liu, et al. Microspore structure formation and transformation of PAN-based carbon fiber during preparation [J]. Materials Review, 2014, 28(9):74-78 (in Chinese). 